\title{
Chemical Treatment with Fluoroquinolones on the Tear Film and Ocular Surface after Cataract Surgery
}

\author{
AURELIAN MIHAI GHITA ${ }^{1 *}$, ANA CRISTINA GHITA ${ }^{2}$, SANZIANA ISTRATE ${ }^{1}$ \\ FLORIN BALTA ${ }^{1}$, RADU CIULUVICA ${ }^{3}$
}

${ }^{1}$ Carol Davila University of Medicine and Pharmacy Faculty of Medicine, Department of Physiology II, 8 Eroii Sanitari Blvd., 050474, Bucharest, Romania

${ }^{2}$ Ocularcare Eye Clinic, Ophthalmology, 128 Ion Mihalache Blvd., 011204, Bucharest, Romania

${ }^{3}$ Carol Davila University of Medicine and Pharmacy, Faculty of Dentistry, Department of Anatomy, 8 Eroii Sanitari Blvd., 050474, Bucharest, Romania

\begin{abstract}
Quinolone antibacterial activity is related to its chemical structure, a synthetic fluorinated analog of nalidixic acid. Cataract surgery is responsible for visual improvement having a major positive impact on the quality of patients' lives. According to the international guidelines, the administration of levofloxacin after cataract surgery decreases the incidence of postoperatively endophthalmitis. In this context we aimed to study the impact of adding topical fluoroquinolones to the conventional treatment after cataract surgery on the ocular surface and tear film. 125 of eyes of 125 patients with cataract were included. The enrolled patients were divided into three groups, a control group of patients that received topical Tobramycin, Dexamethasone and Indomethacin, a second group where moxifloxacin was added and a third group with levofloxacin instead of moxifloxacin. For all the patients, tear film breakup time, Schirmer II test, slit lamp examination preoperatively and postoperatively at one week were performed together with individual Ocular Surface Disease Index at one week after cataract surgery. The test results revealed that $52 \%$ of our studied patients had an abnormal preoperatively tear film breakup time, and $76.8 \%$ after cataract surgery, with a statistically significant decrease of the measured values. The preoperatively measured quantity of tears presented a no statistically significant decrease one week after cataract surgery. Also, we found an increase in the percentage of patients with punctate epithelial erosions. It is important and useful to identify prior to surgery patients with ocular surface disease and the ones at risk to develop clinical manifestations in order to treat them accordingly because, cataract surgery exacerbates the preexistent ocular symptoms. The addition of topical fluoroquinolones after cataract surgery in patients with dry eye is safe, with minimal alterations of the ocular surface and tear production, without statistically significant differences as compared to the control group.
\end{abstract}

Keywords: cataract surgery, topical fluoroquinolones, dry eye disease.

\section{Introduction}

Cataract surgery is one of the most common ocular surgical procedure performed mainly on the aging population[1]. The number of cataract extractions is constantly growing, due to different factors such as increasing of life expectancy, lifestyle, patient mentality and easier access to the latest surgical techniques that allow fast recovery with excellent results. Although cataract surgery is considered an ambulatory routine procedure, potential serious complications resulting in permanent visual impairment can occur. Among these, the development of postoperatively endophthalmitis as a result of an intraocular infection is well-known [2]. Various operative and nonoperative measures have been advocated to prevent this serious complication [2,3]. Preoperative periocular skin disinfection with topical povidone iodine is widely adopted and is considered one of the basic procedures in the prevention of infectious postoperative endophthalmitis[4]. Based on the recent prospective studies, it seems that the administration of intracameral antibiotic at the end of cataract surgery is an effective

\footnotetext{
*email:mihai.ghita@ocularcare.ro
} 
approach in reducing the risk of endophthalmitis. [5-8] There are no well-controlled high-quality studies able to show a benefit after preoperative or postoperative topical antibiotics administration in the prevention of postoperatively endophthalmitis. In the literature the results of different prospective studies indicate that the associations of topical antibiotics perioperatively decrease the microbial growth from periocular samples [9].

According to the international guidelines, such as ESCRS (European Society of Cataract and Refractive Surgery) guidelines for prevention and treatment of endophthalmitis, we have chosen to study fluoroquinolones as antimicrobial agents, administrated topically. Levofloxacin (2S)-7-fluoro- 2methyl-6-(4-methylpiperazin-1-yl) -10-oxo-4-oxa-1-azatricyclo [7.3.1.05,13] trideca-5(13),6,8,11tetraene-11-carboxylic acid) and moxifloxacin (7-[(4aS,7aS)-1,2,3,4,4a,5,7,7a- octahydropyrrolo [3,4b]pyridin-6-yl]-1-cyclopropyl-6-fluoro-8-methoxy-4-oxoquinoline-3-carboxylic acid) have a quinolone molecular structure and the main target is DNA gyrase on which they act through several mechanisms offered by the residues from the positions 1, 3, 4, 6, 7, and 8. [10] (Figure 1).
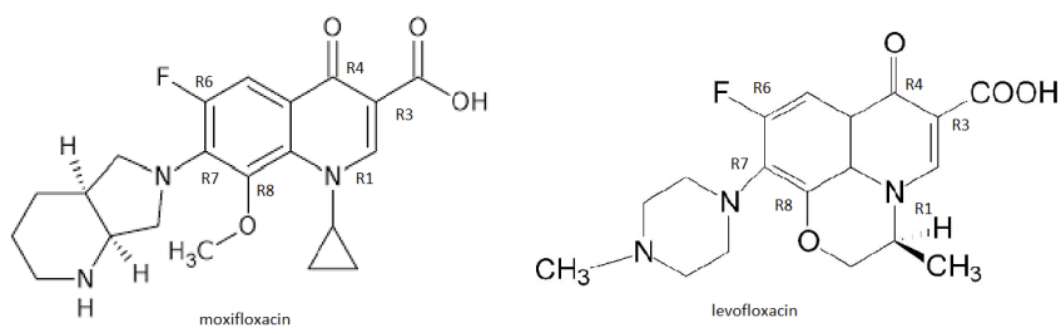

Figure 1. Chemical structure of levofloxacin and moxifloxacin

The residue from position 1 is able to bind due to a hydrophobic interaction to the pocket of enzyme -DNA complex. The presence in this position of cyclopropyl residue for moxifloxacine and metal residue in levo position for levofloxacin and are some of the most potent modification for antimicrobial activity [11]. Positions 3 and 4 play a critical role in the cleavage or modification of the DNA, being essential [12]. The addition of flour in position 6 increases significantly the antimicrobial activity. The nitrogen heterocycle residue from position 7 is considered to be capable of directly binding to DNA gyrase. [11] Adding a residue in position 8 is responsible for the molecular stearic configuration. By their structure, levofloxacin and moxifloxacin have the highest affinity for DNA gyrase, with a strong antimicrobial activity.

The rationale behind the use of preoperative and postoperative antibiotics is to reduce the bacterial load on the ocular surface. Also, the antibiotic levels must exceed the minimal inhibitory concentration (CMI) 90. Studies have shown that ocular instillation of Levofloxacin or other fluoroquinolones (fql) does not reach the needed concentration level [13].

Given topically after cataract surgery, steroidal and non-steroidal anti-inflammatory treatment reduces ocular inflammation providing fast recovery of vision and decreases symptoms but with significantly alteration of the ocular surface [14]. The use of preoperative and/or postoperative fluoroquinolones or other therapeutic methods (such as contact lens)[15] can alter the tear production which can lead to minimal ocular surface alterations $[13,16]$. In this context, we decided to perform a qualitative and quantitative analysis of the tears and ocular surface taking into account the patient's symptomatology after cataract surgery according to the latest guidelines of endophthalmitis prevention.

\section{Materials and methods}

We included patients with cataract without other associated ocular pathology and with a nonoperated congener eye. Patients were excluded if they were noncompliant, did not gave their informed 
consent for performing cataract surgery and if they were on systemic medication or local chronic treatments that could alter the tear production or ocular surface.

125 eyes of 125 patients were included in the study; the patients were evaluated preoperatively and postoperatively at one week after cataract extraction. Patients were divided into one of the three groups. The control group was composed of 37 patients that received postoperative topical treatment with Tobramycin 3mg, Dexamethasone $1 \mathrm{mg}$ and Indomethacin 0.1\%, 1 drop 4 times-a-day. The moxifloxacin group, consisting of 36 patients were moxifloxacin 1 drop 4 times-a-day was added to the previous treatment and the levofloxacin group made of 52 patients, were moxifloxacin was replaced by levofloxacin 1 drop 4 times-a-day. Visual acuity (VA), tonometry, TBUT (Tear break-up time), Schirmer II test, slit-lamp examination and calculation of individual OSDI (Ocular Surface Disease Index) were performed pre and postoperatively at one week (Table 1).

Table 1. Mean and standard deviation of visual acuity, intraocular pression (IOP), OSDI test, Schirmer II test and TBUT before and one week after cataract surgery in each study group and in the whole group of enrolled patients

\begin{tabular}{|c|c|c|c|c|c|c|c|c|}
\hline & \multicolumn{2}{|c|}{ Levofloxacin Group } & \multicolumn{2}{c|}{ Moxifloxacin Group } & \multicolumn{2}{c|}{ Control Group } & \multicolumn{2}{c|}{ Total } \\
\hline & Mean & $\begin{array}{c}\text { Std. } \\
\text { Deviation }\end{array}$ & Mean & $\begin{array}{c}\text { Std. } \\
\text { Deviation }\end{array}$ & Mean & $\begin{array}{c}\text { Std. } \\
\text { Deviation }\end{array}$ & $\begin{array}{c}\text { Std. } \\
\text { Mean }\end{array}$ & \\
\hline $\begin{array}{c}\text { 1 week after } \\
\text { surgery }\end{array}$ & & & & & & & & \\
\hline VA (Log Mar) & 0.10 & 0.14 & 0.09 & 0.12 & 0.06 & 0.07 & 0.09 & 0.12 \\
\hline IOP (mmHg) & 16.81 & 2.93 & 17.64 & 3.50 & 16.92 & 2.02 & 17.08 & 2.88 \\
\hline OSDI test & 10.15 & 9.17 & 9.63 & 6.32 & 16.95 & 12.38 & 12.01 & 10.04 \\
\hline $\begin{array}{c}\text { Schirmer II } \\
\text { test(mm) }\end{array}$ & 7.67 & 4.66 & 7.31 & 4.97 & 7.57 & 5.39 & 7.54 & 4.94 \\
\hline TBUT(s) & 3.31 & 2.55 & 4.14 & 2.47 & 2.95 & 1.45 & 3.44 & 2.29 \\
\hline $\begin{array}{c}\text { Before } \\
\text { Surgery }\end{array}$ & & & & & & & & \\
\hline AV (Log Mar) & 1.09 & 0.71 & 1.03 & 0.57 & 1.40 & 0.91 & 1.16 & 0.75 \\
\hline IOP (mmHg) & 15.27 & 2.73 & 14.08 & 3.98 & 14.41 & 2.49 & 14.67 & 3.01 \\
\hline OSDI test & 26.91 & 16.74 & 29.22 & 18.90 & 39.85 & 22.38 & 31.41 & 19.82 \\
\hline $\begin{array}{c}\text { Schirmer II test } \\
\text { (mm) }\end{array}$ & 9.29 & 6.00 & 9.06 & 4.63 & 9.32 & 4.92 & 9.23 & 5.28 \\
\hline TBUT (s) & 4.87 & 3.07 & 5.89 & 2.80 & 4.46 & 2.42 & 5.04 & 2.85 \\
\hline
\end{tabular}

\section{Results and discussions}

The enrolled patients presented a homogenous distribution of age, between 70 and 75 years old, and a heterogenous distribution of sex in control and moxifloxacin groups (71\% women and $29 \%$ men) and relatively homogenous in Levofloxacin group (52\% women and $48 \%$ men).

After cataract surgery, the visual acuity was significantly improved $(\mathrm{p}<0.001)$ in all study groups from 0.97-1.08 log Mar to 0.07-0.12 log Mar, without any differences between the three groups. Also, in all study groups intraocular pression raised with 1-2 $\mathrm{mmHg}$, with no difference between the groups.

In all studied groups there was a decrease in the lacrimal tear basal secretion but without any statistical significance. There is a statistically significant tear instability in all three groups, before and after cataract surgery $(\mathrm{p}<0.01)$. There are no statistical differences between the studied groups as regarding Schirmer II test and TBUT values that present a mean decrease of 1.45-1.76 mm (Figure 2) and of 1.51-1.75 s respectively in all groups (Figure 3). 


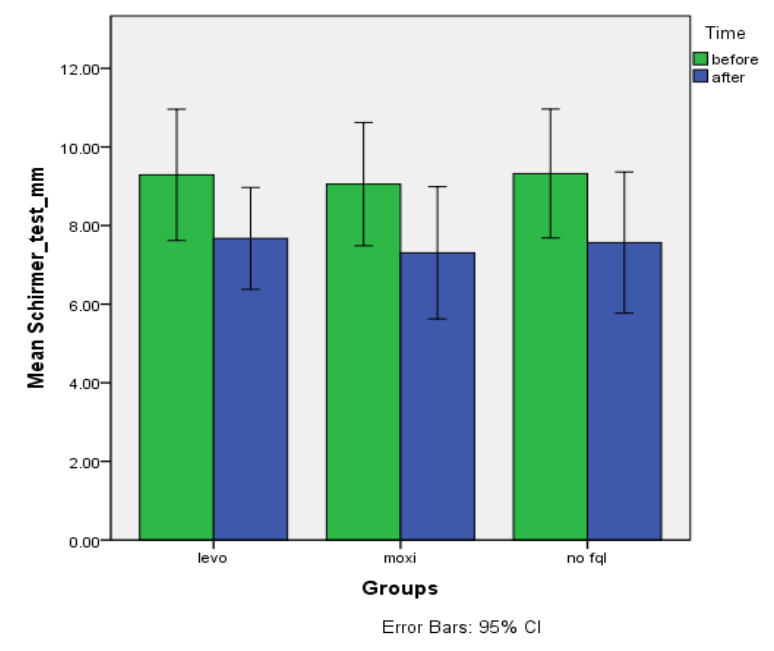

Figure 2. Schirmer II test before and one week after cataract surgery in all three groups (levofloxacin group - levo, moxifloxacin group - moxi, control group - no fql)

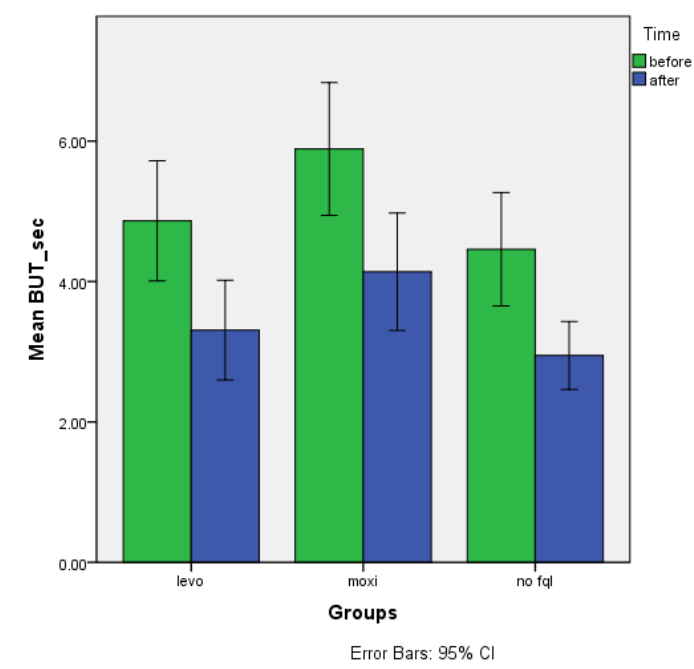

Figure 3. TBUT before and 1 week after cataract surgery

in all three groups (levofloxacin group - levo, moxifloxacin group - moxi, control group - no fql)

$52 \%$ of our studied patients had low values of the tear film breakup time, less than $5 \mathrm{~s}$, before cataract surgery and $76.8 \%$ after cataract surgery. In all three groups we have noticed that a great part of the patients with normal TBUT $(>=10 \mathrm{~mm}$ ) before cataract surgery develop tear film instability (59 s) one week after the surgery, as patients diagnosed with tear film instability develop aspects of dry eye disease (DED). The fact that Moxifloxacin seems to affect less the ocular surface is highlighted by a small percentage of patients with abnormal TBUT results, of $66 \%$ as related with the other study groups respectively, control $91 \%$ and levofloxacin group, 73\%. (Figure 4). In Levofloxacin group we identified a slightly raise in the number of patients, from $59.5 \%$ before to $63.3 \%$ after cataract surgery, that presented a drop in the quantity of the measured tears, under $10 \mathrm{~mm}$. In control group, the number of patients with moderate dry eye $(5-9 \mathrm{~mm})$ decreased at $16.2 \%$ from $48.6 \%$ and the number of patients with severe dry eye (under $5 \mathrm{~mm}$ ) raised to $43 \%$ from $16.2 \%$. (Figure 5) Also, in the moxifloxacin group, the percentage of patients with severe dry eye raised from $5.5 \%$ before the surgery to $30.5 \%$ after the procedure. 

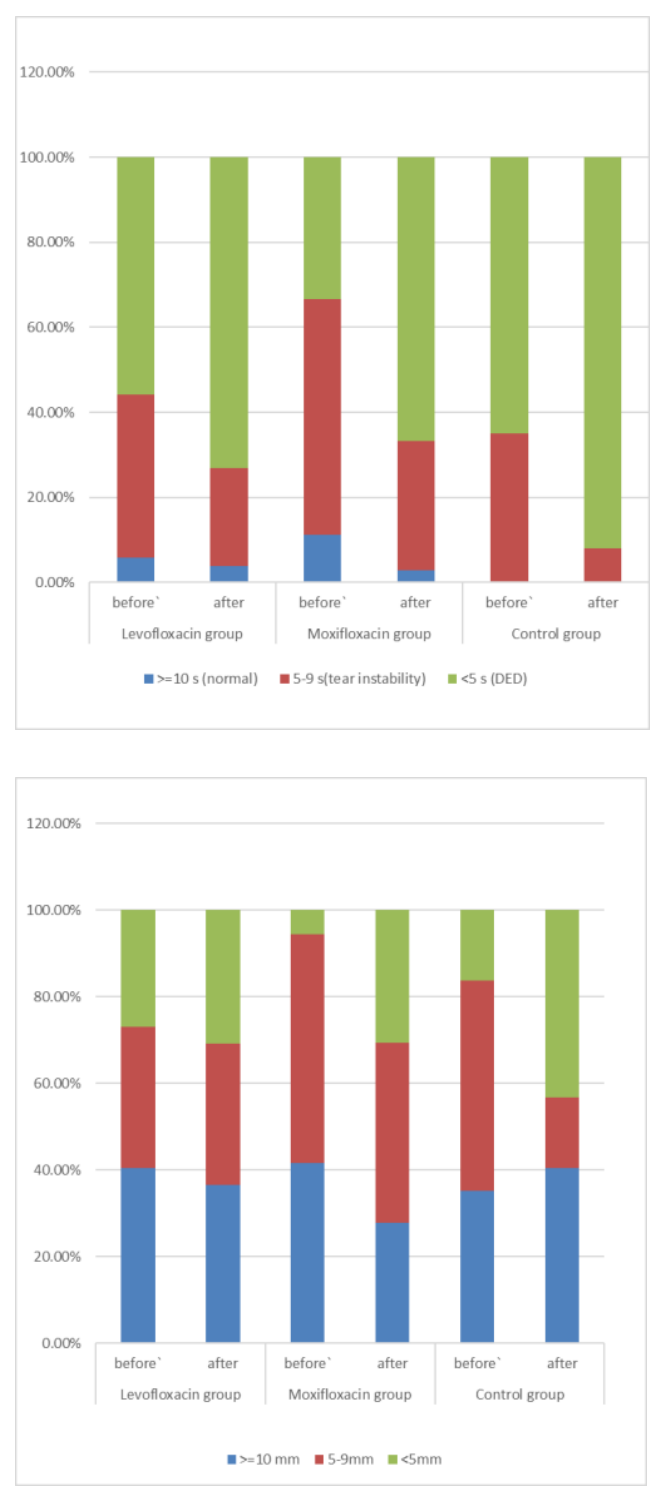

Figure 4. The percentage of patients with TBUT under $5 \mathrm{~s}$, between 5-9 s and above $10 \mathrm{~s}$, before and one week after cataract surgery in all study groups
Figure 5. The percentage of patients with Schirmer II Test under $5 \mathrm{~mm}$, between 5-9 $\mathrm{mm}$ and above $10 \mathrm{~mm}$ at $5 \mathrm{~min}$ before and one week after cataract surgery in all study groups.

If we focus our attention on the slit lamp examination of the ocular surface, we can observe that the incidence of corneal punctate epithelial erosions (PEE) has increased significantly $(p<0.05)$ in all three groups from $20 \%$ to $39.20 \%$ but were very well tolerated, probably due to a decreased sensitivity and by the use of anti-inflammatory drugs (Figure 6).

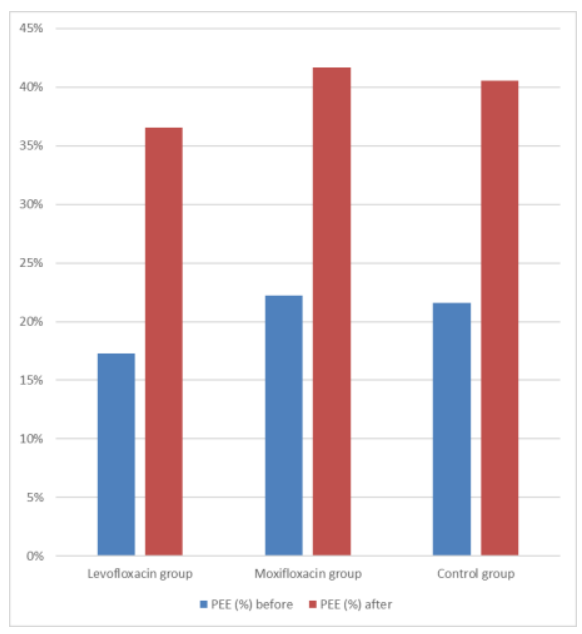

Figure 6. The percentage of patients with PEE before and one week after cataract surgery in all study groups 
Finally, we performed for each patient, ocular surface disease index (OSDI) in the attempt to evaluate and understand better the ocular surface symptomatology. Despite the increase in the incidence of punctate epithelial erosions and the decrease in the quality and quantity of the tears, OSDI score has improved due to visual acuity improvement.

Cataract surgery increases the quality of life especially by improving the visual function. Although cataract surgery is generally considered a safe procedure resulting in a favorable visual outcome, surgical complications do occur. The most feared complication is postoperative endophthalmitis because the visual outcome is often very poor. In accordance with ESCRS study, 70\% of patients had a final visual acuity of 20/200 or less. Treatment of endophthalmitis requires hospital admission and further surgeries [17]. A good correlation between perioperative aseptic measures and prophylactic treatment with intracameral and topical antibiotics peri and post-operative decreases significantly the incidence of endophthalmitis. Patients who received topical treatment with levofloxacin $0.5 \%$ showed a reduction in the rate of endophthalmitis from 2.1 per 1000 patients to 1.5 per 1000 patients but without significantly $[13,16]$.

In this context we have decided to evaluate the tears, ocular surface and symptomatology of the patients as related to cataract surgery, following the newest guidelines of prophylaxis and treatment of endophthalmitis, aiming to establish the impact of topical fluoroquinolones treatment. After cataract surgery topical steroidal and non-steroidal anti-inflammatory drugs are already routinely being associated. Thus, a control group was formed. In the other two groups, moxifloxacin or levofloxacin were added. Like other performed studies, our work revealed important changes in the tear film and ocular surface after cataract surgery due to ocular trauma and topical treatment. After cataract surgery the percent of people with sever DED and a Schirmer II test value under $5 \mathrm{~mm}$ at 5 minutes, increased from $17.6 \%$ before surgery to $34.4 \%$ one week after surgery $\mathrm{p}<0.01$. Many of the patients diagnosed with DED at one week after the surgery were previously considered with tear film instability having a TBUT between 5 to 9 seconds. However, the addition of fluoroquinolones didn't reveal important alterations on the ocular surface and tear film, the changes found in the fluoroquinolones study groups as compared with the control group are not statistically significant.

\section{Conclusions}

It is of great importance to proper identify, prior to cataract surgery, the patients at high risk to develop a sever DED because its presence generates the limitation of visual potential and ocular comfort. It is also important to identify the patients already with ocular surface signs and symptoms in order to treat them accordingly, because cataract surgery exacerbates the preexistent ocular symptoms of dry eye.

The addition of fluoroquinolones after cataract surgery is beneficial because its use is safe, it induces minimal alterations of the ocular surface and tear production, without significant differences as compared to the control group. Judging by the OSDI results, fluoroquinolones are well tolerated by the patients without significant differences between the three studied groups. It is certified that the administration of fluoroquinolones perioperatively and in association with Cefuroxime intracameral, represents a good association in the prevention of endophthalmitis.

Acknowledgments: All authors have contributed equally; No financial disclosure.

\section{References}

1. BROWNE M. The nurse's role in helping patients cope with sight loss. Nurs Times 2003; 99:30-32.

2 .KAMALARAJAH S, SILVESTRI G, SHARMA N et al. Surveillance of endophthalmitis following cataract surgery in the UK. Eye (Lond) 2004; 18:580-587.

3. GHITA AC, GHITA AM, NOAGHI M, POPA CA. The corticosteroids effect on corneal endothelial cell in pulse therapy, specific to the cataract surgery. J Med Life 2014; 7 Spec No. 4:46-53. 
4. NANAVATY MA, WEARNE MJ. Perioperative antibiotic prophylaxis during phaco-emulsification and intraocular lens implantation: national survey of smaller eye units in England. Clin Exp Ophthalmol 2010; 38:462-466.

5. FINTELMANN RE, NASERI A. Prophylaxis of postoperative endophthalmitis following cataract surgery: current status and future directions. Drugs 2010; 70:1395-1409.

6. GARCIA-SAENZ MC, ARIAS-PUENTE A, RODRIGUEZ-CARAVACA G, BANUELOS JB. Effectiveness of intracameral cefuroxime in preventing endophthalmitis after cataract surgery Ten-year comparative study. J Cataract Refract Surg 2010; 36:203-207.

7. RODRIGUEZ-CARAVACA G, GARCIA-SAENZ MC, VILLAR-DEL-CAMPO MC, ANDRESALBA Y, ARIAS-PUENTE A. Incidence of endophthalmitis and impact of prophylaxis with cefuroxime on cataract surgery. J Cataract Refract Surg 2013; 39:1399-1403.

8. GARCIA-SAENZ MC, ARIAS-PUENTE A, RODRIGUEZ-CARAVACA G, ANDRES AY, BANUELOS BJ. [Endophthalmitis after cataract surgery: epidemiology, clinical features and antibiotic prophylaxis]. Arch Soc Esp Oftalmol 2010; 85:263-267.

9.MINO DE KH, KREUTZER TC, AGUIRRE-ROMO I et al. A prospective randomized study to determine the efficacy of preoperative topical levofloxacin in reducing conjunctival bacterial flora. Am J Ophthalmol 2008; 145:136-142.

10. L.R.PETERSON, Quinolone Molecular Structure-Activity Relationships: What We Have Learned about Improving Antimicrobial Activity, Clinical Infectious Diseases 33, no. 2001, p. S180-S186.

11. LLORENTE B, LECLERC F, CEDERGREN R. Using SAR and QSAR analysis to model the activity and structure of the quinolone-DNA complex. Bioorg Med Chem 1996; 4:61-71.

12.TILLOTSON GS., Quinolones: structure-activity relationships and future predictions., J Med Microbiol 44, no. 1996, p. 320-324.

13. SEAL DV, BARRY P, GETTINBY G et al. ESCRS study of prophylaxis of postoperative endophthalmitis after cataract surgery: Case for a European multicenter study. J Cataract Refract Surg 2006; 32:396-406.

14. CONGDON NG, SCHEIN OD, VON KP, LUBOMSKI LH, GILBERT D, KATZ J. Corneal complications associated with topical ophthalmic use of nonsteroidal antiinflammatory drugs. J Cataract Refract Surg 2001; 27:622-631.

15 COSTIN ALEXANDRU LEASU BRANET SILMVEVRCRIABEUFB, Fluoro-siloxanyl Styrene Rigid Gas Permeable Contact Lens, Fabric characteristics and outcomes with extended use on animal model cornea, Revista de Chimie (Rev.Chim.) 70, no. 2019, p. 4003-4006.

16. BARRY P, SEAL DV, GETTINBY G, LEES F, PETERSON M, REVIE CW. ESCRS study of prophylaxis of postoperative endophthalmitis after cataract surgery: Preliminary report of principal results from a European multicenter study. J Cataract Refract Surg 2006; 32:407-410.

17. BARRY P, GARDNER S, SEAL D et al. Clinical observations associated with proven and unproven cases in the ESCRS study of prophylaxis of postoperative endophthalmitis after cataract surgery. J Cataract Refract Surg 2009; 35:1523-31, 1531.

Manuscript received: 26.03 .2020 